\title{
Diagnósticos de enfermería más frecuentes en un centro obstétrico de la ciudad de Guayaquil-Ecuador 2018.
}

Nursing diagnoses more frequent in obstetric center of the city of GuayaquilEcuador 2018.

Lic. Joicy Anabel Franco Coffré. Mg. ${ }^{1}$, Lic. Lina Graciela Calderón Intriago Mg. ${ }^{2}$, Lic. Maira Cristina Cujilán Alvarado Mg. ${ }^{3}$, \& Lic. Jessica Patricia Salazar Menéndez Mg. ${ }^{4}$

\begin{abstract}
.
DOI: https://doi.org/10.33262/cienciadigital.v2i2.81

The most frequent nursing diagnoses in a specific area, facilitate the realization of standardized nursing care plans. Objective Identify the most frequent nursing diagnoses in an obstetric center in Guayaquil city. A quantitative cross-sectional study involving 93 pregnant women or those in the immediate puerperium stage in a third-level hospital care institution in Guayaquil city during the month of January to February 2018. Data collection was carried out through application of the Nursing care process: analysis of clinical history, interview and physical examination, and the use of the NANDA taxonomy. Risk of alteration of the maternal-fetal dyad, risk of bleeding and pain were the most frequent diagnostic labels in the obstetric area. Further studies are expected on the diagnostic accuracy in the obstetric area and validation of such diagnoses.
\end{abstract}

Keywords: Obstetric Nursing, Nursing Process, Nursing Diagnosis

\section{Resumen.}

Los diagnósticos de enfermería que son más frecuentes en un área específica, facilitan la realización de planes de cuidado de enfermería estandarizados. Identificar los

${ }^{1}$ Universidad de Guayaquil, Carrera de Enfermería, Guayaquil Ecuador, joicy.francoc@ug.edu.ec

${ }^{2}$ Universidad de Guayaquil, Carrera de Enfermería, Guayaquil Ecuador, lina.calderoni @ug.edu.ec

${ }^{3}$ Universidad de Guayaquil, Carrera de Enfermería, Guayaquil Ecuador, maira.cujilana@ug.edu.ec

${ }^{4}$ Universidad de Guayaquil, Carrera de Enfermería, Guayaquil Ecuador, jessica.salazarm@ug.edu.ec 
diagnósticos de enfermería más frecuentes en un centro obstétrico de la ciudad de Guayaquil. Estudio cuantitativo de tipo transversal que involucró a 93 mujeres gestantes o en etapa de puerperio inmediato en una institución hospitalaria de tercer nivel de atención de la ciudad de Guayaquil durante el mes de enero a febrero del 2018. La recolección de datos se realizó mediante aplicación del proceso de atención de enfermería: análisis de historia clínica, entrevista y examen físico, y la utilización de la taxonomía NANDA. Riesgo de la alteración de la diada materno-fetal, Riesgo de sangrado y Dolor fueron las etiquetas diagnosticas de mayor frecuencia en el área obstétrica. Se esperan estudios adicionales sobre la exactitud diagnóstica en el área obstétrica y validación de tales diagnósticos.

Palabras Claves: Enfermería obstétrica; Proceso de enfermería; Diagnóstico de enfermería.

\section{Introducción.}

La muerte materna se refiere a la muerte de una mujer mientras está embarazada o dentro de los 42 días siguientes a la terminación del embarazo, debida a cualquier causa, sea relacionada con la gestación, su atención o causas accidentales e incidentales ${ }^{1}$. En el Ecuador durante los últimos años se han visto datos alarmantes con relación a la mortalidad materna. La Razón de mortalidad materna (RMM) el año 1990 fue de 154 por 100.000 nacidos vivos ${ }^{2}$, para el 2014 fue de 49.16 por cada 100.000 nacidos vivos ${ }^{2}$, reduciendo en un $68 \%$ durante todo este periodo. Entre las causas de la RMM en el Ecuador, se encuentran con un 35.83 las Causas Obstétricas directas, en segundo lugar, la Hemorragia posparto con un valor de 8 , y en tercer lugar la Eclampsia ${ }^{2}$.

Según el Ministerio de Salud Pública del Ecuador (MSP), la mayoría de las muertes maternas se producen en mujeres que viven en las zonas rurales o urbanas marginales que por lo general poseen un nivel de pobreza alto, con inaccesibilidad a los servicios básicos a las vías de acceso y unidades educativas ${ }^{3}$. Como dato adicional, en el Ecuador hay 8'087.914 mujeres. De ellas 1'069.988 son jefas de hogar. El 55.5\% son mujeres en edad fértil entre 15 y 49 años según la Encuesta Nacional de Salud y Nutrición ${ }^{4}$, siendo uno de los grupos prioritarios y más importantes dentro de la población ecuatoriana y al mismo tiempo la más vulnerable.

Ante este contexto, la atención en salud que se debe brindar a este grupo poblacional, debe ser oportuna, protocolizada y concienzuda. En el Ecuador, existe una Componente normativo materno neonatal elaborado por el MSP donde se encuentran los protocolos y estándares en la atención de la mujer embarazada y en estado de puerperio, así como en el neonato ${ }^{5}$. Contiene información teórica sobre la patología, el tratamiento específico, esquemas terapéuticos y flujogramas de atención, no obstante, la implicación de enfermería en el mismo es casi inexistente. 
El proceso de atención de enfermería (PAE) consiste en la aplicación del método científico y sistemático de intervenciones para la implementación de cuidados profesionales en los pacientes sanos o enfermos, familia o comunidad ${ }^{6}$. La enfermera, con una visión holística del ser humano procede a la valoración del paciente mediante la recolección de datos tanto objetivos como subjetivos. Objetivos como hallazgos encontrados en el examen físico, pruebas de laboratorio o imágenes etc., y datos subjetivos providenciados por el paciente en la realización de la entrevista.

Respetando la individualidad de la persona, la enfermera mediante razonamiento multifacético y toma de decisiones complejas ${ }^{7}$, respetando la individualidad de la persona, protocoliza sus intervenciones o cuidados, de acuerdo a la construcción del diagnóstico enfermero (respuestas humanas reales, de riesgo, o de bienestar) ${ }^{8}$ y visualiza una meta o resultado (Nursing Outcomes Classifications) ${ }^{9}$ y las intervenciones que permitirán llegar a ese resultado (Nursing Interventions Classifications) ${ }^{10}$, el plasmar todo estos elementos de forma escrita se denomina plan de cuidado ${ }^{11}$.

En las áreas hospitalarias gineco-obstétricas, se observa una dinámica muy común y hasta cierto punto repetitivo. Mujeres con amenaza de aborto, abortos espontáneos, abortos retenidos, embarazo molar, embarazos de riesgo, trastornos hipertensivos del embarazo entre otras, donde la atención de enfermería debe ser acuciosa, ágil y de conocimientos específicos de la terapéutica a seguir. Por ello y mediante el análisis de la importancia de la actuación de enfermería en la atención de esta población vulnerable, se realiza este estudio que tuvo como objetivo general, determinar cuáles fueron los diagnósticos de enfermería más frecuentes en un área ginecoobstetrica para poder visibilizar cuales serían a partir de ellos, las intervenciones de enfermería a realizarse y poder proponer planes de cuidado de enfermería estandarizados.

\section{Método.}

Investigación de enfoque cuantitativo, de tipo descriptivo y corte transversal. La muestra estuvo conformada por 93 pacientes que llegaban al área de emergencia obstétrica, posparto (ya sea en puerperio fisiológico o puerperio quirúrgico) y hospitalización de obstetricia o también denominada Alojamiento conjunto de un hospital del Seguro Social de la ciudad de Guayaquil- Ecuador (Hospital Ceibos) durante 45 días. Fueron pacientes captadas al azar y cuyos criterios de inclusión se basaron en poseer complicaciones del embarazo o encontrarse en el puerperio quirúrgico o fisiológico inmediato.

Como criterios de exclusión mujeres con patologías ginecológicas u otras que no tengan que ver con el embarazo. Se elaboró un formato de valoración de enfermería en salud reproductiva focalizada utilizando los patrones funcionales de Marjorie Gordon ${ }^{12}$, para la recolección de datos, y aplicación del pensamiento crítico en la elección del diagnóstico enfermero prioritario que sería asignado a cada situación de las pacientes. Adicionalmente, 
se analizaron las historias clínicas de las pacientes para constatar los datos subjetivos providenciados en la entrevista.

Posterior a la elección de la etiqueta diagnostica se elaboró una matriz de sistematización donde fueron colocadas variables como edad, diagnóstico médico, datos objetivos reales, datos subjetivos reales, y diagnóstico de enfermero asignado, para posteriormente determinar la prevalencia de los diagnósticos e enfermería más frecuentes. Como parte de la ética, se hizo una solicitud al área hospitalaria para realizar la recolección de datos en las historias clínicas de las pacientes como el acceso a las habitaciones para la valoración de enfermería al pie de cama y la firma de consentimiento informado de las pacientes.

\section{Resultados.}

Al finalizar la organización de los datos de las 93 mujeres, quedaron distribuidas por edad, la tabla 1 especifica que la el 30,10\% de la muestra correspondió a mujeres con edades comprendidas entre 29-33 años, y en menor medida un 4\% de adolescentes.

Tabla 1. Edades de las pacientes del centro obstétrico.

\begin{tabular}{lll}
\hline Edad & Frecuencia & Porcentaje \\
\hline $\mathbf{1 5 - 1 8}$ años & 4 & $4 \%$ \\
$\mathbf{1 9 - 2 3}$ años & 15 & $16,12 \%$ \\
$\mathbf{2 4 - 2 8}$ años & 24 & $25,80 \%$ \\
$\mathbf{2 9 - 3 3}$ años & 28 & $30,10 \%$ \\
$>\mathbf{3 4}$ años & 22 & $23,65 \%$ \\
\multicolumn{1}{c}{ Total } & 93 & $100 \%$ \\
\hline
\end{tabular}

Fuente: Elaboración propia.

El diagnóstico médico más frecuente fue el parto único por cesárea con un 25,80\%, y los menos frecuentes el aborto espontaneo, embarazo ectópico, trombocitopenia secundaria, oligohidramnios, verrugas venéreas anogenitales, y la anemia que complica el embarazo, parto y puerperio. Los diagnósticos médicos fueron agrupados bajo la nominación del CIE10 , en la tabla 2 .

Tabla 2. Diagnósticos médicos del cie-10 de las pacientes.

\begin{tabular}{llll}
\hline Código & Diagnóstico médico basado en CIE -10 & No & $\%$ \\
\hline O82 & Parto único por cesárea & 24 & $25,80 \%$ \\
O42.0 & $\begin{array}{l}\text { Ruptura prematura de las membranas: trabajo de parto durante las } \\
\text { primeras 24 horas }\end{array}$ & 15 & $16,12 \%$ \\
$\mathbf{O 1 4 . 0}$ & Preeclampsia moderada & 10 & $10,75 \%$ \\
O60 & Parto prematuro & 8 & $8,60 \%$ \\
O42.1 & $\begin{array}{l}\text { Ruptura prematura de las membranas: trabajo de parto después de las } \\
\text { primeras 24 horas }\end{array}$ & $4,30 \%$ \\
O86.2 & Infección vías urinarias consecutiva al parto & 4 & $4,30 \%$
\end{tabular}




\begin{tabular}{llll} 
O44 & Placenta previa & 4 & $4,30 \%$ \\
OUT.4 & Histerectomía & 4 & $4,30 \%$ \\
O14.1 & Preeclampsia severa & 3 & $3,22 \%$ \\
O13 & $\begin{array}{l}\text { Hipertensión gestacional (inducida por el embarazo) } \\
\text { significativa }\end{array}$ & sin proteinuria & 3 \\
O02.1 & Aborto Retenido & $3,22 \%$ \\
O05.4 & Otro Aborto incompleto sin complicación & 2 & $2,15 \%$ \\
O47 & Falso trabajo de parto & 2 & $2,15 \%$ \\
O45 & Desprendimiento prematuro de placenta & 2 & $2,15 \%$ \\
O03 & Aborto espontaneo & 2 & $2,15 \%$ \\
O00 & Embarazo ectópico & 1 & $1,07 \%$ \\
D69.5 & Trombocitopenia secundaria & 1 & $1,07 \%$ \\
O41.0 & Oligohidramnios & 1 & $1,07 \%$ \\
A63.0 & Verrugas veneras anogenitales & 1 & $1,07 \%$ \\
O99.0 & Anemia que complica el embarazo, parto y puerperio & 1 & $1,07 \%$ \\
TOTAL & 1 & $1,07 \%$ \\
\hline
\end{tabular}

Fuente: Elaboración propia.

En base a la valoración de enfermería a cada paciente realizada, y mediante el proceso de atención de enfermería, se eligieron las etiquetas diagnosticas referentes a las respuestas humanas reales (tabla 3) se obtuvo el 49,68\%, de riesgo (tabla 4) o de bienestar (tabla 5) que se encontraron en el momento de la valoración, las cuales se especifican en la tabla 3.

Tabla 3. Etiquetas diagnósticas de enfermería reales.

\begin{tabular}{lll}
\hline Etiquetas diagnósticas Reales & No & $\%$ \\
\hline Dolor & 10 & $10,75 \%$ \\
Dolor de parto & 6 & $6,45 \%$ \\
Perfusión tisular ineficaz & 5 & $5,37 \%$ \\
Ansiedad & 3 & $3,22 \%$ \\
Deterioro de la eliminación urinaria & 3 & $3,22 \%$ \\
Lactancia materna ineficaz & 3 & $3,22 \%$ \\
Afrontamiento ineficaz & 2 & $2,15 \%$ \\
Exceso de volumen de líquidos & 2 & $2,15 \%$ \\
Deterioro de la movilidad física & 2 & $2,15 \%$ \\
Interrupción de la lactancia materna & 2 & $2,15 \%$ \\
Intolerancia a la actividad & 1 & $1,07 \%$ \\
Proceso de maternidad ineficaz & 1 & $1,07 \%$ \\
Déficit de volumen de líquidos & 1 & $1,07 \%$ \\
Privación del sueño & 1 & $1,07 \%$ \\
Gestión ineficaz de la propia salud & 1 & $1,07 \%$ \\
Incontinencia urinaria de esfuerzo & 1 & $1,08 \%$
\end{tabular}




\begin{tabular}{lll} 
Deterioro de la integridad cutánea & 1 & $1,07 \%$ \\
Leche materna insuficiente & 1 & $1,07 \%$ \\
$\begin{array}{l}\text { Total, pacientes con diagnósticos } \\
\text { reales }\end{array}$ & 46 & $49,38 \%$ \\
Total diagnósticos reales & 18 & \\
\hline
\end{tabular}

Fuente: Elaboración propia.

Predominó la etiqueta diagnostica Dolor, en segundo lugar, la etiqueta de Dolor de parto, las que fueron menos frecuentes correspondieron a intolerancia a la actividad, proceso de maternidad ineficaz, déficit de volumen de líquidos, gestión ineficaz de la propia salud, incontinencia urinaria de esfuerzo entre otras. Los diagnósticos de enfermería reales estuvieron presentes en un $49,38 \%$

Tabla 4. Etiquetas diagnósticas de enfermería de riesgo

\begin{tabular}{lll}
\hline Etiquetas diagnósticas de Riesgo & No & \multicolumn{1}{c}{} \\
\hline Riesgo de alteración de la diada materno fetal & 17 & $18,27 \%$ \\
Riesgo de sangrado & 13 & $13,97 \%$ \\
Riesgo de infección & 9 & $9,67 \%$ \\
Riesgo de hemorragia & 1 & $1,07 \%$ \\
Riesgo de desequilibrio de la temperatura corporal & 1 & $1,07 \%$ \\
Riesgo de proceso de maternidad ineficaz & 1 & $1,07 \%$ \\
TOTAL DE PACIENTES CON DIAGNÓSTICOS DE RIESGO & $\mathbf{4 2}$ & $\mathbf{4 5 , 1 6 \%}$ \\
TOTAL DE DIAGNÓSTICOS DE RIESGO & $\mathbf{6}$ & \\
\hline
\end{tabular}

Fuente: Elaboración propia.

El riesgo de alteración de la diada materno fetal, fue la etiqueta diagnostica de enfermería más frecuente y subsecuentemente la etiqueta Riesgo de sangrado y riesgo de infección. Los diagnósticos de enfermería de Riesgo estuvieron presentes en la muestra de estudio en un $45,16 \%$

Tabla 5. Etiquetas diagnósticas de enfermería de bienestar.

\begin{tabular}{lcll}
\hline Etiquetas diagnósticas de Bienestar & No & \% \\
\hline Disposición para mejorar el proceso de maternidad & 3 & $3,22 \%$ \\
Disposición para mejorar los conocimientos & 1 & 1,07 \\
Disposición para mejorar la lactancia materna & 1 & 1,07 \\
TOTAL DE PACIENTES CON DIAGNÓSTICOS DE & 5 & $5,37 \%$ \\
BIENESTAR & \multicolumn{3}{c}{3} \\
TOTAL DE DIAGNÓSTICOS DE BIENESTAR & \multicolumn{3}{c}{} \\
\hline
\end{tabular}

Fuente: Elaboración propia. 
Las etiquetas diagnósticas de Bienestar fueron las menos frecuentes. Entre las que se obtuvieron fueron: Disposición para mejorar el proceso de maternidad, disposición para mejorar los conocimientos, y disposición para mejorar la lactancia materna. Los diagnósticos de enfermería de bienestar fueron los menos presentes en la muestra de estudio con un $5,37 \%$.

\section{Discusión.}

La importancia de ejecutar este tipo de investigaciones facilita la elaboración de protocolos de atención de enfermería específicos al área de trabajo en la que se desenvuelven los cuidados. No se ha encontrado en la búsqueda de las bases de datos científicas información de diagnósticos de enfermería frecuentes en un área obstétrica, los hay sí, en pacientes diabéticos e hipertensos donde un $98.9 \%$ correspondió al manejo ineficaz de la salud ${ }^{13}$, diagnósticos de enfermería en el área perioperatorio ${ }^{14}$, en ancianos hospitalizados ${ }^{15}$ y en pacientes con disturbios respiratorios ${ }^{16}$, aproximándose un poco más, una investigación en un área ginecológica17 y que por ende posee respuestas humanas diferentes a las de un área obstétrica.

El parto único por cesárea $(25.80 \%)$ sobre el parto vaginal $(20,42 \%)$ se contrasta con el valor estadístico del Instituto Ecuatoriano de estadísticas y censos (INEC) que manifestó que hasta el 2015 el 50,3\% de los nacidos vivos nacieron por un parto normal, y el 47,8\% por cesárea $^{18}$, esto como último dato de esta institución. El parto por cesárea en el contexto de esta investigación, se dio en su mayoría por la desproporción cefalopélvica, pre eclampsia, o cicatriz uterina previa.

Entre los diagnósticos de enfermería de Riesgo de alteración de la diada materno fetal y perfusión tisular ineficaz se encuentran inmersos los diagnósticos médicos de pre eclampsia y amenaza de parto pre término (en el CIE 10 como parto prematuro), ya que se consideraba una mala perfusión a aquella gestante con hipertensión arterial y también así mismo a la gestante que tenía contracciones uterinas antes de las 37 semanas de gestación y con dilatación cervical, esta ambivalencia podría haber conllevado un valor erróneo en cuanto a la elección de la etiqueta diagnostica de enfermería.

En el caso de los diagnósticos de enfermería de riesgo, predominaron el riesgo de infección, riesgo de sangrado, que fueron relacionadas con el diagnostico medico de ruptura prematura de membranas y el riesgo de sangrado con la placenta previa oclusiva total. Fueron muy bien identificados y son etiquetas de la taxonomía NANDA que no se encuentran en el dominio sexualidad reproducción y que sin embargo tienen mucha injerencia en la problemática obstétrica.

Como limitación principal de este estudio, se encuentra el componente humano que valoro y eligió la etiqueta diagnostica para la construcción del diagnóstico de enfermería. El enfermero realiza un análisis holístico del ser humano, tanto en la entrevista de enfermería 
como en el examen físico, recolección de datos de la historia clínica entre otras informaciones más, y si bien el proceso puede aplicarse de manera óptima, podría haber dentro de esta investigación un sesgo del evaluador al conocer las etiquetas diagnosticas que ofrece la NANDA en la sección del Dominio Sexualidad-reproducción, llegando a pensar que si están en este dominio la gestante debe tener etiquetas de dicha sección, esto provocaría entonces, la eliminación de posibilidades de elección de otras etiquetas que se encuentran en los demás dominios.

Otra limitación del estudio, tiene que ver con el hecho de que las pacientes se encontraron en tres áreas diferentes: emergencia obstétrica, área de monitorización y pre anestesia, y alojamiento conjunto. En cada una de estas áreas las respuestas humanas difieren por el momento que vive la gestante. En un área de emergencia, existe ansiedad, dolor, hasta afrontamiento ineficaz, mientras que en el alojamiento conjunto la preocupación de alimentar a su bebe ante un mal agarre del pezón, daría como diagnóstico de enfermería la lactancia materna ineficaz. Por ello se recomendaría que este tipo de estudios sean desarrollados en áreas separadas para poder tener un resultado más concreto y especifico.

\section{Conclusiones.}

- El identificar las respuestas humanas reales, de riesgo, de bienestar, y sindromicas de un área de salud determinada, facilita la creación de protocolos estandarizados de cuidados de enfermería basados en las taxonomías NANDA, NIC Y NOC. El lenguaje estandarizado de enfermería facilita la documentación, investigación, visibilidad del enfermero y ejecución de los cuidados en una población específica.

- Los diagnósticos de enfermería reales estuvieron presentes en un 49,38\%, los de riesgo en un 45,16\%, y los diagnósticos de enfermería de bienestar fueron los menos presentes en la muestra de estudio con un 5,37\%, lo cual deja en consideración que, los diagnósticos de enfermería de bienestar podrían ser considerados como menos importantes y no ser tomados en cuenta durante una hospitalización obstétrica.

- En el área obstétrica predominaron diagnósticos de enfermería tales como Riesgo de alteración de la diada materno fetal, riesgo de sangrado y Dolor, cuyos contextos médicos son enfermero-dependientes al $100 \%$, ya que necesitan monitoreo y control permanente enfermeril como el principal cuidador de las mujeres de embarazo de riesgo o cuando ya se encuentran en el trabajo de parto. Los líderes de servicios obstétrico debiesen poder enfocar dicha problemática con mayor detenimiento y acuciosidad, para así poder beneficiar al país mediante el descenso de la mortalidad materna en sus estadísticas, y más allá de eso, el poder salvaguardar el binomio madre-hijo de cualquier situación que atente contra su bienestar. 


\section{Referencias bibliográficas.}

Suárez González J, Gutiérrez Machado M, Lorenzo González B. Problemática de la muerte materna en Villa Clara. [Online]. 2012 [cited 2018 Febrero 20. Available from: http://scielo.sld.cu/scielo.php?script=sci_arttext\&pid=S0138600X2012000100004\& Ing=es

Instituto Nacional de Estadística y Censos. [citado 20 de febrero de 2018]. Disponible en: http://www.ecuadorencifras.gob.ec/vdatos/

Ministerio de Salud Pública del Ecuador, Plan Nacional de Salud Sexual y Salud Reproductiva: Viceministerio de Gobernanza de la Salud Pública, Marzo, 2017, Quito, Ecuador.

Freire WB., Ramírez-Luzuriaga MJ., Belmont P., Mendieta MJ., Silva-Jaramillo MK., Romero N., Sáenz K., Piñeiros P., Gómez LF., Monge R. (2014). Tomo I: Encuesta Nacional de Salud y Nutrición de la población ecuatoriana de cero a 59 años. ENSANUT-ECU 2012. Ministerio de Salud Pública/Instituto Nacional de Estadísticas y Censos. Quito-Ecuador.

Ministerio de Salud Pública del Ecuador, Componente normativo materno, Consejo Nacional de salud, agosto, 2008, Quito, Ecuador.

Díaz Aguilar R., Amador Morán R., Alonso Uría R.M., Campo González A., Mederos Dueñas M., Oria Saavedra M. Evaluación del conocimiento del Proceso de Atención de Enfermería: Estudiantes de licenciatura de Enfermería. Facultad "Lidia Doce". 2013. Enferm. univ [revista en la Internet]. 2015 Mar [citado 2018 Feb 27]; 12(1):36-40. Disponible en: http://www.scielo.org.mx/scielo.php?script=sci_arttext\&pid=S1665$70632015000100006 \& \operatorname{lng}=\mathrm{es}$.

Lambie A, Schwend K, Scholl A. Utilization of the Nursing Process to Foster Clinical Reasoning During a Simulation Experience. SAGE Open. 2015 Dec 23;5(4):2158244015617516.

Herdman TH, editora. NANDA International. Diagnósticos Enfermeros. Definiciones y clasificación. 2015-2017. Barcelona: Elsevier; 2015

Moorhead S. Jonson M. Maas M. Clasificación de resultados de enfermería (NOC). $5^{a}$ Ed. Madrid: Elsevier España; 2013. 
Bulechek G, Buthcer H, McCloskey J. Clasificación de intervenciones de enfermería (NIC). 6a Ed. Madrid: Elsevier España; 2013

Hernández LC, Graña León MC, González Montero L, Santana del Río M, Miranda Guerra Ad. Plan de cuidados de Enfermería estandarizado para asesoramiento genético de embarazadas con alfafetoproteina alterada en Caibarien. Revista Cubana de Enfermería [revista en Internet]. 2016 [citado 2018 Feb 27];32(3): [aprox. 0 p.]. Disponible en: http://www.revenfermeria.sld.cu/index.php/enf/article/view/1330

Gordon M, Manual de diagnósticos enfermeros. 10ª ed. España: Elsevier; 2003

Sampaio F de C, Oliveira PP de, Mata LRF da, Moraes JT, Fonseca DF da, Vieira VA de S. Perfil de diagnósticos de enfermería en personas hipertensas y diabéticas. Invest Educ Enferm. 201706;35(2):139-53

Nakasato GR, Lopes CT, Lopes J de L, Barros ALBL de. Diagnósticos de enfermagem no perioperatório de cirurgia cardíaca. REME rev min enferm. 201512;19(4):980-6

Araújo DD de, Carvalho RLR de, Chianca TCM. Nursing diagnoses identified in records of hospitalized elderly. Invest Educ Enferm. 201407;32(2):225-35.

Santod NA dos, Cavalcante TF, Lopes MV de O, Gomes EB, Oliveira CJ de. Perfil de diagnósticos de enfermería en pacientes con disturbios respiratorios. Invest Educ Enferm. 201504;33(1):112-8.

Lopes MHB de M, Montagnoli ÉT de L, Maia CAT. Diagnósticos de enfermagem mais freqüentes em uma unidade de internação de ginecologia. Revista Latino-Americana de Enfermagem. diciembre de 1999;7(5):77-84.

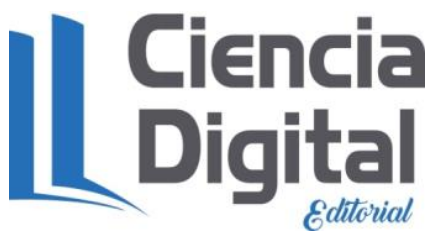


Para citar el artículo indexado.

Franco J., Calderón L., Cujilán M. \& Salazar J. (2018). Diagnósticos de enfermería más frecuentes en un centro obstétrico de la ciudad de Guayaquil-Ecuador 2018. Revista electrónica Ciencia Digital 2(2), 170-180. Recuperado desde: http://cienciadigital.org/revistacienciadigital2/index.php/CienciaDigital/article/view/81/75

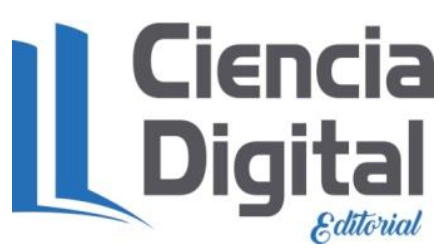

El artículo que se publica es de exclusiva responsabilidad de los autores y no necesariamente reflejan el pensamiento de la Revista Ciencia Digital.

El articulo queda en propiedad de la revista y, por tanto, su publicación parcial y/o total en otro medio tiene que ser autorizado por el director de la Revista Ciencia Digital.
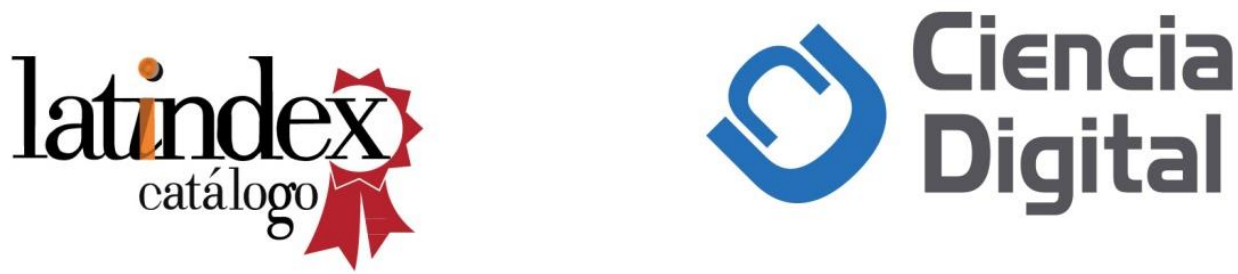